\title{
ANÁLISE COMPARATIVA DA FREQÜÊNCIA DE PREMATURIDADE E BAIXO PESO ENTRE FILHOS DE MÃES ADOLESCENTES E ADULTAS
}

\section{COMPARATIVE ANALYSIS OF PREMATURITY AND LOW BIRTH WEIGHT BETWEEN NEWBORNS OF ADOLESCENT AND ADULT MOTHERS}

Cristina Mika Suzuki*

Maria Esther Jurfest Ceccon ${ }^{* *}$

Mario Cícero Falcão***

Flávio Adolfo Costa Vaz ${ }^{* * * *}$

Suzuki CM, Ceccon MEJ, Falcão MC, Vaz FAC. Análise comparativa da frequência de prematuridade e baixo peso entre filhos de mães adolescentes e adultas. Rev Bras Crescimento Desenvolv Hum. 2007; 17(3): 95-103.

\begin{abstract}
Resumo: A gravidez na adolescência é um problema de saúde pública por ser considerada uma gravidez de alto risco. A incidência de prematuridade e de baixo peso ao nascer entre filhos de mães adolescentes varia dependendo da região e pais estudado. Objetivo: realizar uma análise comparativa da freqüência de prematuridade e baixo peso entre filhos de mães adolescentes e adultas. Método: estudo prospectivo comparativo de uma série de casos no qual foram incluídos 132 mães e seus recém nascidos (51 mães adolescente e 81 mães adultas) internados em dois hospitais públicos da cidade de São Paulo, no período de junho de 2005 a maio de 2006. Em relação às mães foram analisadas as seguintes variáveis: idade, raça, escolaridade, ocupação, número de gestações e de abortos, pré-natal (número de consultas), antecedentes mórbidos antes e durante o pré-natal, tipo de parto e uso de drogas lícitas e ilícitas. Em relação aos recémnascidos foram analisados: Boletim de Apgar, sexo, peso de nascimento, idade gestacional, adequação nutricional e aleitamento materno. Resultados: A gravidez na adolescência esteve relacionada com nascimentos de crianças prematuras (16-31.4\% no grupo de mães adolescentes e 7-8.65\% no grupo de mães adultas, $\mathrm{p}=0.01$ ), e esta relação não foi observada com o baixo peso ao nascer (14-27.5\% no grupo adolescente e 16-19.8\% no grupo de mães adultas, $\mathrm{p}=0.17$ ). Não houve diferenças significantes em relação aos outros parâmetros. Conclusões: a assistência prénatal pareceu diminuir a freqüência de baixo peso ao nascer em filhos de mães adolescentes. Não houve interferência na freqüência da prematuridade.
\end{abstract}

Palavra-chave: Gravidez. Adolescência. Recém-nascido. Baixo peso. Prematuridade.

\section{INTRODUÇÃO}

Gravidez e maternidade são complemen- tações do desenvolvimento humano racional, linear e com possibilidades de planejamento. Sendo assim, a gravidez na adolescência produz efei-

\footnotetext{
* Programa de Iniciação Científica. Departamento de Pediatria da Faculdade de Medicina da Universidade de São Paulo.

** Livre Docente em Pediatria pela Faculdade de Medicina da Universidade de São Paulo. Médica responsável pela Unidade de Cuidados Intensivos Neonatal do Instituto da Criança do HCFMUSP e coordenadora do Berçário do Hospital Estadual de Sapopemba-HC

*** Doutor em Pediatria pela Faculdade de Medicina da Universidade de São Paulo. Médico assistente da Unidade de Cuidados Intensivos Neonatal do Instituto da Criança do Hospital das Clínicas da Faculdade de Medicina da Universidade de São Paulo

**** Professor Titular do Departamento de Pediatria. Chefe da Disciplina de Pediatria Neonatal do Instituto da Criança do Hospital das Clínicas da Faculdade de Medicina da Universidade de São Paulo.
} 
tos deletérios sobre o desenvolvimento biológico e psíquico, com possíveis efeitos prejudiciais à inserção na vida social.

Em vários países a gravidez na adolescência é considerada o maior problema de saúde pública referente a essa faixa etária. Os fatores responsáveis por tal fato são: redução da idade da menarca; maior liberdade sexual com diminuição da idade para início da vida sexualmente ativa; falha na educação sexual, implicando em falta de conhecimentos sobre concepção e a escassez de serviços de planejamento familiar ${ }^{1}$.

A maternidade na adolescência, freqüentemente não planejada e indesejada, é referida como um impacto negativo nas condições físicas, emocionais e econômicas das adolescentes, afetando completamente seu modo de vida. ${ }^{2} \mathrm{~A}$ maioria das adolescentes abandona os estudos para cuidar da criança, ocorrendo aumento dos riscos de desemprego, mudança de estrato sócio-econômico e dependência econômica dos familiares, ${ }^{3}$ perpetuando-se assim, a pobreza, educação limitada, abuso e violência familiar tanto à mãe quanto à criança. ${ }^{4}$

A gravidez na adolescência é considerada de alto risco, uma vez que ela está sujeita a maior incidência de complicações na gestação, parto e puerpério, intensificando-se ainda mais quando a mãe pertence a uma classe social menos favorecida, consequiências estas que podem aumentar a incidência de prematuridade e baixo peso ao nascer ${ }^{2,5-8}$.

Assim, condições sanitárias precárias e falta de acesso ou ausência de recursos de saúde para acompanhamento pré-natal podem contribuir para um acréscimo no risco reprodutivo, prejudicando tanto a mãe quanto a criança.

A morbimortalidade infantil ainda é elevada em países em desenvolvimento, principalmente entre os filhos de mães adolescentes ${ }^{9}$. Devido ao baixo poder aquisitivo e acesso restrito aos recursos de saúde, as adolescentes grávidas não recebem o apoio necessário para acompanhar a gestação nem orientação quanto aos cuidados básicos a fim de garantir o bem estar da criança ${ }^{2}$. Assim, deixa-se de salientar, por exemplo, a importância da amamentação, e conseqüentemente, pode-se observar maior mortalidade in- fantil, principalmente por doenças gastrintestinais e respiratórias ${ }^{9}$.

Considerando-se as repercussões negativas que a falta de assistência pode acarretar tanto à saúde da mãe adolescente quanto à de seu filho, a realização de um estudo comparativo entre mães adolescentes e adultas possibilitaria verificar a existência de diferenças entre estes dois grupos principalmente em relação ao nascimento de recém-nascidos prematuros e/ou de baixo peso ao nascer. Os resultados deste estudo poderão nos direcionar para ações de saúde que visem a melhorias no atendimento a este grupo de pacientes.

O objetivo é realizar uma análise comparativa da freqüência de prematuridade e baixo peso ao nascer entre filhos de mães adolescentes e adultas.

\section{MÉTODO}

Trata-se de um estudo prospectivo de uma série de casos, cujos dados foram coletados entre agosto de 2005 e maio de 2006, após aprovação do Comitê de Ética em Pesquisa do Instituto da Criança do Hospital das Clínicas da Faculdade de Medicina da Universidade de São Paulo.

Foram incluídos no estudo recém-nascidos de mães adolescentes e de mães adultas internados no Berçário do Hospital Estadual de Sapopemba do Hospital das Clínicas da Faculdade de Medicina da Universidade de São Paulo e na Unidade de Cuidados Intensivos Neonatal do Instituto da Criança, também do Hospital das Clínicas da Faculdade de Medicina da Universidade de São Paulo, São Paulo.

Foram excluídos da pesquisa os recémnascidos portadores de síndromes genéticas, malformações congênitas, afecções neurológicas congênitas, bem como nascimentos múltiplos.

A prematuridade foi definida pela idade gestacional inferior a 37 semanas e o baixo peso por peso de nascimento inferior a $2500 \mathrm{~g}$, conforme critérios da Organização Mundial da Saúde $(1977)^{10}$.

Para o cálculo da idade gestacional foram utilizadas, por ordem de prioridade:

- informação materna sobre a data da úl- 
tima menstruação (Regra de Naegele, que considera o tempo de gestação normal de 280 dias), quando esta diferir em no máximo duas semanas da idade gestacional fornecida pela ultra-sonografia fetal realizada até a $20^{\mathrm{a}}$ semana da gestação;

- ultrassonografia realizada até 20 semanas de gestação ${ }^{11}$, nos casos em que a idade gestacional materna não foi considerada confiável e a diferença entre as idades calculadas pelos métodos ultra-sonográfico e pós-natal foi inferior a duas semanas;

- idade gestacional pós-natal calculada através do Método de New Ballard ${ }^{12}$ para prematuros e Capurro ${ }^{13}$ para recém-nascidos de termo, quando estes diferirem em mais de duas semanas das idades gestacionais materna e ultra-sonográfica.

A adequação nutricional dos recém-nascidos foi baseada nas curvas de referência de peso para a idade gestacional obtidas por Alexander e colaboradores (1996) ${ }^{14}$, sendo que foram considerados adequados para a idade gestacional os recém-nascidos situados entre os percentis 10 e 90 da curva, grandes para a idade gestacional aqueles situados acima do percentil 90 e pequenos para a idade gestacional aqueles situados abaixo do percentil 10 .

Os seguintes dados foram obtidos por entrevista com as mães, após o nascimento: escolaridade, ocupação e utilização de fumo, álcool e drogas ilícitas, tanto das mães quanto dos pais. Por consulta aos prontuários maternos foram levantados os seguintes dados: cor, número de gestações e abortamentos, realização de pré-natal e número de consultas, antecedentes maternos e gestacionais e tipo de parto. Em relação ao recém-nascido foram selecionados: sexo, Boletim de Apgar, peso de nascimento, idade gestacional, adequação nutricional e aleitamento materno na ocasião da alta hospitalar do neonato.

Dentre os recém-nascidos que preencheram os critérios de inclusão e exclusão durante o período de estudo houve uma perda amostral de, aproximadamente, $16 \%$. Esta perda se justifica pela impossibilidade dos pesquisadores estarem presentes para a coleta de dados do questionário ou pela ausência do pai da criança durante o período de internação do binômio mãe-filho.

Para a análise estatística os resultados foram analisados de modo univariado através de comparações entre adolescentes e adultas no que concerne a cada possível fator de risco. Para a comparação entres as médias utilizou-se o teste $t$ de Student e para as proporções, o teste Quiquadrado, pelo programa Sigma Stat. Para a significância foi considerado um valor de $\mathrm{p}$ inferior a 0,05 .

\section{RESULTADOS}

O estudo foi composto por 132 recémnascidos (51 filhos de mães adolescentes e 81 filhos de mães adultas).

\section{DISCUSSÃO}

Este estudo teve como objetivo principal demonstrar a influência da idade materna no nascimento de prematuros e recém-nascidos de baixo peso. Entretanto, analisaram-se também outros elementos, como as características sócioeconômicas dos pais, para uma melhor compreensão da problemática apresentada.

A idade média das mães adolescentes foi de 17,3 anos e das mães adultas de 26,8 anos, sendo que neste uma mãe tinha 42 anos; não houve diferença entre mães de cor branca e não branca.

Em relação à escolaridade: nível fundamental incompleto e completo, médio incompleto e completo, superior incompleto e completo observou-se que todas as adolescentes tinham algum grau de escolaridade e entre as adultas, 2 $(2,5 \%)$ não tinham nenhum grau. Nenhuma mãe adulta tinha curso superior. Houve diferença estatisticamente significante $(\mathrm{p}=0,038)$ em relação ao estudo médio incompleto, sendo que as mães adultas já tinham completado o ensino médio e apenas 4 adolescentes tinham terminado este ciclo.

Em relação à ocupação, as mães adolescentes eram do lar ou estudantes enquanto as adultas tinham alguma ocupação, com diferença significativa $(\mathrm{p}=0,04)$. Além disso, observou-se que $14(27,5 \%)$ fumavam, $2(3,9 \%)$ ingeriam ál- 
Tabela 1: Características maternas de ambos os grupos do estudo

\begin{tabular}{l|lll}
\hline & $\begin{array}{l}\text { Adolescentes } \\
\mathbf{n}=\mathbf{5 1}\end{array}$ & $\begin{array}{l}\text { Adultas } \\
\mathbf{n}=\mathbf{8 1}\end{array}$ & Valor de p \\
\hline Idade (anos) & $17,3 ? 1,3$ & $26,8 ? 5,6$ & $\mathrm{p}<0,001$ \\
Cor & $29(56,9 \%)$ & $50(61,7 \%)$ & $\mathrm{p}=0,89$ \\
$\quad$ Branca & $22(43,1 \%)$ & $31(38,3 \%)$ & $\mathrm{p}=0,84$ \\
Não branca & 0 & & \\
Escolaridade & $20(39,2 \%)$ & $23(28,4 \%)$ & $\mathrm{p}=0,46$ \\
Nenhuma & $6(11,8 \%)$ & $12(14,8 \%)$ & $\mathrm{p}=0,86$ \\
EF incompleto & $21(41,2 \%)$ & $14(17,3 \%)$ & $\mathrm{p}=0,038$ \\
EF completo & $4(7,8 \%)$ & $30(37,0 \%)$ & $\mathrm{p}=0,06$ \\
EM incompleto & 0 & 0 & \\
EM completo & 0 & 0 & \\
ESUP incompleto & & & \\
ESUP completo & $22(43,2 \%)$ & $40(49,4 \%)$ & $\mathrm{p}=0,79$ \\
Ocupação & $25(49 \%)$ & 0 & \\
Do lar & 0 & $9(11,1 \%)$ & \\
Estudante & $4(7,8 \%)$ & $32(39,5 \%)$ & $\mathrm{p}=0,04$ \\
Doméstica & $14(27,5 \%)$ & $16(19,8 \%)$ & $\mathrm{p}=0,17$ \\
Outras & $5(35,7 \%)$ & $4(25,0 \%)$ & $\mathrm{p}=0,71$ \\
Fumo & $2(3,9 \%)$ & $9(11,1 \%)$ & $\mathrm{p}=0,3$ \\
? 10cigarros/dia & $2(3,9 \%)$ & $1(1,2 \%)$ & $\mathrm{p}=0,7$ \\
Álcool & & & \\
Drogas ilícitas & & & \\
\hline & & & \\
\hline
\end{tabular}

EF: ensino fundamental; EM: ensino médio; ESUP: ensino superior

Tabela 2: Antecedentes gestacionais das mães de ambos os grupos do estudo

\begin{tabular}{l|lll}
\hline & $\begin{array}{l}\text { Adolescentes } \\
\mathbf{n}=\mathbf{5 1}\end{array}$ & $\begin{array}{l}\text { Adultas } \\
\mathbf{n}=\mathbf{8 1}\end{array}$ & Valor de p \\
\hline Número de gestações & $38(74,5 \%)$ & $20(24,7 \%)$ & $\mathrm{p}=0,01$ \\
1 & $11(21,6 \%)$ & $25(30,9 \%)$ & $\mathrm{p}=0,48$ \\
2 & $2(3,9 \%)$ & $15(18,5 \%)$ & $\mathrm{p}=0,056$ \\
3 & 0 & $21(25,9 \%)$ & \\
4 ou mais & $2(3,9 \%)$ & $15(18,5 \%)$ & $\mathrm{p}=0,056$ \\
Número de abortamentos & $17(33,3 \%)$ & $26(32,1 \%)$ & $\mathrm{p}=0,94$ \\
Doenças maternas & $4(7,8 \%)$ & $1(1,2 \%)$ & $\mathrm{p}=0,16$ \\
ITU & $1(2,0 \%)$ & 0 & \\
DHEG & $6(11,8 \%)$ & $10(12,3 \%)$ & $\mathrm{p}=0,85$ \\
DG & $4(7,8 \%)$ & $7(8,6 \%)$ & $\mathrm{p}=0,86$ \\
Leucorréia & $21(41,2 \%)$ & $42(51,9 \%)$ & $\mathrm{p}=0,57$ \\
Outras & $49(96,1 \%)$ & $81(100 \%)$ & $\mathrm{p}=0,97$ \\
Nenhuma & $6,5(3,1 \%)$ & $6,7(2,8 \%)$ & $\mathrm{p}=0,7$ \\
Seguimento pré-natal & & & $\mathrm{p}=0,57$ \\
\hline Número de consultas no Pré-natal & & & \\
Tipo de parto & $36(70,6 \%)$ & $69(85,2 \%)$ & $\mathrm{p}=0,15$ \\
Vaginal & $15(29,4 \%)$ & $12(14,8 \%)$ & \\
Operatório & & & \\
\hline
\end{tabular}

ITU: infecção do trato urinário; DHEG: doença hipertensiva da gravidez; DG: diabetes gestacional 
Tabela 3: Características dos recém-nascidos de ambos os grupos do estudo

\begin{tabular}{|c|c|c|c|}
\hline & $\begin{array}{l}\text { Adolescentes } \\
\mathbf{n}=\mathbf{5 1}\end{array}$ & $\begin{array}{l}\text { Adultas } \\
\mathbf{n}=\mathbf{8 1}\end{array}$ & Valor de $p$ \\
\hline \multicolumn{4}{|l|}{ Sexo } \\
\hline Masculino & $22(43,1 \%)$ & $35(43,2 \%)$ & $\mathrm{p}=0,88$ \\
\hline Feminino & $29(56,9 \%)$ & $46(56,8 \%)$ & \\
\hline Peso (gramas) & $2677,7 ? 726,7$ & $3116,7 ? 563,4$ & $\mathrm{p}<0,001$ \\
\hline Prematuridade & $16(31,4 \%)$ & $7(8.65 \%)$ & $\mathrm{p}=0,01$ \\
\hline Baixo peso & $14(27,5 \%)$ & $16(19,8 \%)$ & $\mathrm{p}=0,17$ \\
\hline \multicolumn{4}{|l|}{ Classificação nutricional } \\
\hline PIG & $6(11,8 \%)$ & $6(7,4 \%)$ & $\mathrm{p}=0,64$ \\
\hline AIG & $43(84,4 \%)$ & $71(87,7 \%)$ & $\mathrm{p}=0,91$ \\
\hline GIG & $2(3,9 \%)$ & $4(4,9 \%)$ & $\mathrm{p}=0,86$ \\
\hline Apgar de $5^{\circ}$ minuto $>7$ & $50(92,2 \%)$ & $81(100 \%)$ & $\mathrm{p}=0.78$ \\
\hline \multicolumn{4}{|l|}{ Aleitamento } \\
\hline LM exclusivo & $36(70,6 \%)$ & $75(92,6 \%)$ & $\mathrm{p}=0,38$ \\
\hline Misto & $8(15,7 \%)$ & $5(6,2 \%)$ & $\mathrm{p}=0,19$ \\
\hline Fórmula & $7(13,7 \%)$ & $1(1,2 \%)$ & $\mathrm{p}=0,019$ \\
\hline
\end{tabular}

PIG: pequeno para a idade gestacional; AIG: adequado para a idade gestacional; GIG: grande para a idade gestacional; LM: leite materno

estatisticamente significativa em relação ao uso de drogas nos dois grupos (tabela 1).

Em relação às características paternas, observou-se que as adolescentes têm filhos também com adolescentes e adultos jovens, idade média de 23,3 anos quando comparados com a média dos pais do outro grupo que foi de 31,1 anos, com valor de $\mathrm{p}<0,001$. As variáveis escolaridade e ocupação apresentaram resultados semelhantes às mães. Observou-se que os pais fumam mais e utilizam mais drogas ilícitas que as mães e chama atenção a alta ingestão de álcool nos pais do grupo das mães adultas.

Quando se analisaram os antecedentes gestacionais das 132 mães (tabela 2), verificouse que $100 \%$ das mães adultas e $96 \%$ das mães adolescentes realizaram todas as consultas de pré-natal. Entretanto, como esperado, houve diferença significante em relação às mães adolescentes terem menor número de gestações do que as adultas $(\mathrm{p}=0,01)$. Todas as outras variáveis analisadas não mostraram diferenças estatisticamente significantes (tabela 1).

Em relação às características dos recémnascidos (tabela 3), observou-se diferença estatisticamente significante em relação ao peso de nascimento $(2677,7 \pm 726,7$ nas adolescentes e $3116,7 \pm 563,4$ nas adultas, com $p<0,001$ ), além de maior número de prematuros no grupo de mães adolescentes (16 recém-nascidos - 31,4\% no grupo de adolescentes e $7-8,65 \%$ recém-nascidos no de adultas, com $\mathrm{p}=0,01)$. Entretanto, em relação ao baixo peso ao nascer não se observou diferença significativa (14 recém-nascidos - 27,5\% do grupo de mães adolescentes e 16 recém-nascidos - 19,8\% de mães adultas, com $\mathrm{p}=0,17$ ), assim como também nas outras variáveis estudadas, tais como: tipo de parto, sexo, Boletim de Apgar e adequação nutricional. Na ocasião da alta hospitalar, 70,6 \% dos filhos de mães adolescentes e 92,6\% dos filhos de adultas estavam recebendo aleitamento materno exclusivo.

Em relação aos fatores demográficos, salientou-se a diferença quanto à ocupação das mães adultas, apresentando maior variedade de profissões, ao passo que as mães com idade inferior a 20 anos se restringem à ocupação do lar ou estudante em sua maioria, correspondendo a 92\% das ocupações exercidas pelas adolescentes. Isto se deve à legislação trabalhista em vigor, a qual restringe o vínculo empregatício para 
menores de 18 anos, salvo exceções. Além disso, em relação à escolaridade foi constatada diferença significante quanto à não conclusão do ensino médio. As adolescentes com ensino médio incompleto perfazem quase metade das mães deste grupo, sendo que as que completaram o ensino médio são apenas $7,8 \%$. No grupo das mães adultas, $37 \%$ conseguiram completar todo o ensino médio. Esse dado reflete uma das conseqüências mais prejudiciais da gravidez na adolescência, que é o abandono dos estudos para se dedicar aos cuidados do filho ${ }^{15}$.

Apesar de a adolescência estar associada a comportamentos de risco, neste estudo não se observaram diferenças em relação ao uso de drogas lícitas e ilícitas ${ }^{16}$. As mães adolescentes fumam mais que as adultas, porém apresentam menor taxa de etilismo.

Observou-se também que a maior parte das mães adolescentes eram primíparas e o máximo de gestações encontrado neste grupo foi de três. Nas adultas, as secundíparas foram mais freqüentes, seguidas de quatro ou mais gestações em $25,9 \%$ das mães deste grupo, havendo mulheres cuja gestação era a décima terceira. Verificou-se aqui a necessidade de melhorar os programas de planejamento familiar, pois quanto mais cedo as jovens engravidam, maior o risco de elas abandonarem os estudos e ficarem expostas ao desemprego, perpetuando, assim, a pobreza $^{17,18}$. Além disto, seria desejável a existência de um número maior de programas de educação sexual nas escolas e melhor acesso da população de baixo poder aquisitivo aos métodos contraceptivos com intuito de se evitar a gravidez não planejada ${ }^{19,20}$.

Apesar de $18,5 \%$ das adultas terem tido abortamentos em contraposição a apenas 3,9\% das adolescentes, não houve diferença estatística. A maior freqüência de abortos não provocados no grupo das mães adultas deve-se à idade mais avançada, a qual eleva a probabilidade de uma gravidez complicada.

O acompanhamento pré-natal foi realizado por todas as adultas e por $96,1 \%$ das mais jovens. $\mathrm{O}$ número de consultas foi praticamente semelhante, mostrando que, apesar de não ser o ideal, o sistema básico de saúde vem garantindo o direito de seus cidadãos ao acesso à saúde.

As doenças mais freqüentes durante a gestação, em ambos os grupos foram a infecção do trato urinário e a leucorréia, com incidências semelhantes, tanto em adultas como em adolescentes $^{3,5}$. Estes dois fatores são indutores de parto prematuro, no entanto, como as pacientes receberam tratamento adequado, isto não deve ter tido peso importante na gênese da prematuridade.

O parto vaginal foi predominantemente maior nos dois grupos. Isso se deve à política de incentivo do Sistema Único de Saúde brasileiro em diminuir os partos operatórios, a fim de minimizar os custos para a saúde e otimizar a recuperação das mães. Deve-se lembrar que o Brasil é um dos países com as maiores taxas de cesáreas no mundo e políticas para reverter esta situação são sempre bem-vindas.

Em relação às características dos recémnascidos, não houve diferença em relação ao sexo nos dois grupos. Entretanto, houve diferença estatística quanto ao peso, sendo $2677,7 \mathrm{~g}$ a média do peso de nascimento de recém-nascidos de mães adolescentes e $3116,7 \mathrm{~g}$, a dos filhos de mães adultas. Este fato, no entanto, não refletiu em diferença de baixo peso entre os dois grupos, ou seja, $14(27,5 \%)$ no grupo de mães adolescentes e $16(19,8 \%)$ entre as mães adultas, com $\mathrm{p}=0,17$.

A porcentagem mais elevada de prematuros em mães adolescentes $(31,4 \%)$ contrasta com o estudo realizado por Jimenez e colaboradores $(2000)^{1}$, que não encontraram associação entre gravidez juvenil e prematuridade. Entretanto, os nossos resultados estão em concordância com a pesquisa de Silva e colaboradores $(2003)^{21}$, cuja associação entre idade materna inferior a 20 anos e prematuridade se manteve, mesmo após o ajuste de fatores reprodutivos e sócio-econômicos. Segundo esses autores, a associação entre gravidez na adolescência e prematuridade se relaciona com a primiparidade, seguimento pré-natal inadequado e infecções maternas, principalmente do trato urinário. No estudo aqui apresentado, apesar de não ter apresentado significância estatística, a primiparidade e a infecção do trato urinário foram analisadas como fatores de risco para a prematuridade ${ }^{22}$.

Em relação ao baixo peso, Maritoni e Bar- 
ros Filho ${ }^{7,8}$ concluíram que a gravidez na adolescência não representou risco para o crescimento fetal, mesmo quando a influência de fatores psicossociais, antecedentes gestacionais, exposição ao fumo e cuidados inadequados de prénatal foram afastados, em concordância com o nosso estudo.

Pelo exposto, a associação entre gravidez na adolescência e prematuridade e ou baixo peso ainda não está totalmente estabelecida. Relatos que confirmaram tal associação provavelmente estudaram gravidezes em idades mais precoces, até 14 anos e com precária assistência pré-natal $^{23}$. Por outro lado, os estudos que constataram tal associação, principalmente entre a gestação na adolescência e o baixo peso ao nascer, sugerem que o crescimento intra-uterino deficiente não seria uma causa direta ou determinante independente da gravidez em idade precoce ${ }^{7,8}$.

No que diz respeito aos aspectos sociodemográficos relacionados à gravidez na adolescência, a maioria dos estudos destaca a interferência no percurso acadêmico, em que têm sido verificados altos índices da baixa escolaridade entre mães adolescentes, em decorrência da evasão, abandono e dificuldade de retorno à escola ${ }^{1,5,20}$. Existe consenso sobre diferentes fatores determinantes (estruturais e sociais) que podem interferir no processo de escolarização de jovens ${ }^{24}$.

São múltiplos os fatores que podem influenciar na baixa escolaridade de adolescentes, entretanto, ao longo das três últimas décadas, as pesquisas têm demonstrado que a maternidade precoce pode comprometer o processo de escolarização, principalmente em condições socioeconômicas desfavoráveis.

Vitalle $(2001)^{25}$ destaca que a prematuridade e baixo peso na adolescência não influenciou a ocorrência de baixo peso, porém aumenta em 1,3 vezes o risco de ocorrência de prematuridade. Destaca ainda que a inadequada condição econômica foi o fator de risco mais importante na determinação de prematuridade e baixo peso. A idade materna, tabagismo e cuidados prénatal proveram um risco aumentado de 1,8 vezes de prematuridade e 2,1 vezes de baixo peso ao nascimento quando a parturiente provinha do baixo nível econômico.

A gravidez na adolescência tem sérias implicações biológicas, familiares, emocionais e econômicas, além das jurídico-sociais, que atingem o indivíduo isoladamente e a sociedade como um todo, limitando ou mesmo adiando as possibilidades de desenvolvimento e engajamento dessas jovens na sociedade. Devido às repercussões sobre a mãe e sobre o concepto é considerada gestação de alto risco pela Organização Mundial da Saúde (OMS 1977, 1978), porém, atualmente postula-se que o risco seja mais social do que biológico.

A gravidez na adolescência não deve ser encarada somente pelo lado biológico, pois envolve aspectos mais amplos relacionados ao ser adolescente. Isto implica em uma discussão mais ampla na tentativa de compreender como o processo da gravidez é vivido na adolescência, no sentido de se buscar medidas de prevenção, adiando a maternidade para quando a mulher estiver preparada para ser mãe. Devido às repercussões sobre a mãe e sobre o concepto é considerada gestação de alto risco pela Organização Mundial da Saúde (OMS 1977, 1978), porém, atualmente postula-se que o risco seja mais social do que biológico.

A assistência pré-natal pareceu diminuir a freqüência de baixo peso ao nascer em filhos de mães adolescentes. Não houve interferência na freqüência da prematuridade.

\footnotetext{
Abstract:Adolescent pregnancy is a high-risk gestation and the incidence of prematurity and low birth weight in adolescent mothers' newborns is different in each region and country. Objective: to carry out a comparative analysis of prematurity and low birth weight frequency between newborns of adolescent and adult mothers. Methods: this is a prospective study that investigated 132 mothers and their children (51 adolescent mothers and 81 adult mothers) that were hospitalized in two public hospitals in the city of São Paulo from June, 2005 to May, 2006. The following data were compared: mothers - age, race, school level, occupation, number of gestations and abortions, prenatal follow-up (number of medical visits), type of delivery and use
} 
of drugs. For the neonates: Apgar Score, gender, gestational age, birth weight, nutritional classification and breastfeeding. Results: pregnancy in adolescence was statistically associated with premature births (16-31.4\% in the adolescent group and 7-8.65\% in the adult group, $\mathrm{p}=0.01$ ) but not with low birth weight (14-27.5\% in the adolescent group and 16-19.8\% in the adult group, $\mathrm{p}=0.17)$. There were no significant differences in relation to the other parameters. Conclusions: we observed that an adequate prenatal follow-up seemed to reduce the low birth weight frequency in newborns of adolescent mothers but not the frequency of prematurity.

Keywords: Pregnancy. Adolescence. Newborn. Prematurity. Low birth weight.

\section{REFERENCIAS}

1. Jimenez MAR, Martin AR, Garcia JRF. Comparing the biological and psychosocial risks of pregnancy between groups of adolescents and adults. Eur J Epidemiol 2000; 16: 527-32.

2. Gomes R, Fonseca EMGO, Veiga. AJMO. Rev Latino-am Enfermagem 2002; 10: 408-14.

3. Olausson PO, Haglund B, Weitoft GR, Cnattingius S. Premature death among teenage mothers. Inter J Obstet and Gynaecol 2004; 111: 793-99.

4. Rentschler DD. Pregnant adolescents' perspectives of pregnancy. Am J Matern Child Nurs 2003; 28: 377-83.

5. Fraser AM, Brockert JE, Ward RH. Association of young maternal age with adverse reproductive outcomes. N Engl J Med 1995; 332: 1113-7.

6. Flanagan PJ, McGrath MM, Meyer EC, Coll CTG. Adolescent development and transitions to motherhood. Pediatrics 1995; 96: 273-77.

7. Mariotoni GGB, Barros Filho AA. A gravidez na adolescência é fator de risco para o baixo peso ao nascer? J Pediatr (Rio) 1998; 74: 107-13.

8. Mariotoni GGB, Barros Filho AA. A gravidez na adolescência é fator de risco para o baixo peso ao nascer? (Brasil) Rev Chil Pediatr 2000; 71 : 453-60.

9. Victora CG, Fuchs SC, Flores JA, Fonseca W, Kirkwoos B. Risk factors for pneumonia among children in a Brazilian metropolitan area. Pediatrics 1994; 93: 977-85.

10. WHO: Recommended definitions, terminology and format for statistical tables related to the perinatal period and use of a new certificate for cause of perinatal deaths. Acta Obstet Gynecol Scand 1977; 56: 247-53.

11. Hadlock FP, Deter RL, Harrist RB, Park SK. Estimating fetal age: computer assisted analysis of multiple fetal growth parameters. Radiology
1984; 152: 497-501.

12. Ballard JL, Khoury JC, Wedig K, Wang L, Eilers-Waalsman BL, Lipp R. New Ballard score expanded to include extremely premature infants. J Pediatr 1991; 119: 417-23.

13. Capurro H, Konichezky S, Fonseca D, Caldeyro-Barcia R. A simplified method for diagnosis of gestational age in the newborn infant. J Pediatr 1978; 93: 120-2.

14. Alexander GR, Himes JH, Kaufman RB, Mor J, Kogan M. A United States national reference for fetal growth. Obst Gyn 1996; 87: 163-8.

15. Dallas C. Family matters: how mothers of adolescents parents experience adolescent pregnancy and parenting. Public Health Nurs 2004; 21:347-53.

16. Dewan N, Brabin B, Wood L, Dramond S, Cooper $\mathrm{C}$. The effects of smoking on birthweight-for-gestacional-age curves in teenage and adult primigravidae. Publ Health 2003; 117, 31-5.

17. Spear HJ. A follow-up case study on teenage pregnancy: "havin' a baby isn't a nightmare, but it's really hard". Pediat Nurs 2004, 30: 120-5.

18. Gama SGN, Szwarcwald CL, Leal MC, Theme Filha MM. Gravidez na adolescência como fator de risco para baixo peso ao nascer no município do Rio de Janeiro, 1996 a 1998. Rev Saúde Pública 2001; 35: 74-80.

19. Maehr JC, Lizarraga JL, Wingard, DL, Felice ME. A comparative study of adolescent and adult mothers who intend to breastfeed. J Adolesc Health 1993; 14: 453-57.

20. Dobson JV, Bryce L, Glaeser PW, Losek JD. Age limits and transition of health care in pediatric emergency medicine. Pediatr Emerg Care 2007; 23: 294-7.

21. Silva AAM, Simões VMF, Barbieri M et al. Young maternal age and preterm birth. Pediat Perinatol Epidemiol 2003; 17: 332-39.

22. Stevens-Simon C, Kaplan DW, McAnarney ER. Factors associated with preterm delivery among 
pregnant adolescents. J Adolesc Health 1993, 14: 340-42.

23. Stern C. El embarazo en la adolescencia como problema público: una visión crítica. Salud Publ Mex 1997; 39: 137-43.

24. Costa MCO. Fecundidade na adolescencia: perspectiva histórica e atual. J Pediatr (Rio)
1998; 74:87-90.

25. Vitalle, M.S.S. Adolescência e outros fatores de risco (nível econômico, cuidado pré-natal e tabagismo) como determinantes de prematuridade e baixo peso. São Paulo, 2001. [Tese doutorado. Universidade Federal de São Paulo-Escola Paulista de Medicina], 147p.

Recebido em: 17/10/2007

Modificado em: 26/11/2007 Aprovado em: 02/12/2007 